(C) Journal of Applied Mathematics \& Decision Sciences, 2(2), 193-199 (1998) Reprints Available directly from the Editor. Printed in New Zealand.

Technical Note

\title{
AN INVESTIGATION OF CHAOS IN THE RL-DIODE CIRCUIT USING THE BDS TEST
}

\author{
R. KASAP \\ Department of Statistics, Gazi University, Ankara, Turkey. \\ E. KURT \\ Department of Physics Education, Gazi University, Ankara, Turkey.
}

\begin{abstract}
In this paper, RL-diode circuit driven by a sinusoidal voltage is employed to obtain nonlinear experimental data. The BDS test statistic is used to analyse these data. According to the results of the analysis for the first differenced order data, chaotic structure has been found for each $e$ values.
\end{abstract}

Keywords: Chaos, RL-diode, BDS test.

\section{Introduction}

There can be aperiodic and complex behaviors in many simple deterministic systems, a pendulum, a fluid under convection or some chemical reactions (Gleick, 1987 ; Keener and Tyson, 1986). In 1981, P. A. Linsay from the Massachusetts Institute of Technology carried out the first rigorous study of the chaotic behavior of an electrical circuit (Smith, 1992). Experimentally an electrical circuit possesses a number of advantages over an optical or a chemical system (Newell, et al.., 1996). One such advantage is that the experimenter has control over many of the parameters which influence the behavior. In the past two decades, many observations of chaotic behaviour in electrical circuits have been reported, the Van der Pol oscillator, the RL-diode and Chua's circuits can be mentioned as examples (Hasler, 1987).

An important reason for carrying out a research on nonlinear systems is that they can potentially explain the variations that seem to be random. Scheinkman and LeBaron (1989), and Hsieh (1991) use the BDS statistic (Brock, Dechert, and Scheinkman, 1987) to test for independence in stock market data. Hsieh (1989) uses the BDS statistic to detect non-linearity and chaos in monetary exchange rates. The use of BDS statistic for testing other financial time series data in economics for nonlinear structure is now a fairly well established practice.

In this paper, time series data obtained from a driven RL-diode circuit is tested for chaotic structure using the BDS. This is the first time that the BDS test has been used to identify chaotic structure in electrical studies. 
Section 2 covers a brief explanation of the BDS test. Section 3 displays the results of the experiment on the electrical circuit. Section 4 summarizes the results of the BDS test. Finally, the main findings of this study are presented in section 5 .

\section{The BDS Test}

The BDS approach tests the null hypothesis that the variable of interest is independently and identically distributed (IID). This test is more powerful than the alternative of deterministic chaos or stochastic non-linear models (Brock, et al., 1991). Now, let us briefly consider the test statistics-BDS itself. It is based on the so-called integral introduced by Grasberger and Procaccia (1983).

The time series to be analysed $\left(X_{t}: t=1,2, \ldots, T\right)$ is used to form the so-called $N$-histories

$$
X_{t}^{N}=\left(X_{t}, X_{t+1}, \ldots, X_{t+N-1}\right)
$$

Each $N$-history can be considered to be point in an $N$-dimensional space, where $N$ is called the embedding dimension. These $N$-histories can be used to define a correlation integral

$$
C_{N}(e)=\frac{2}{T_{N}\left(T_{N}-1\right)} \sum_{t<s} \sum I_{e}\left(X_{t}^{N}, X_{s}^{N}\right),
$$

where $T_{N}=T-N+1$, and $I_{e}$ is the indicator function of the event

$$
\left|X_{t+i}-X_{s+i}\right|<e, i=0,1, \ldots, N-1 \text {. }
$$

i.e. $I_{e}\left(X_{t}^{N}, X_{s}^{N}\right)$ is unity if $\left|X_{t}^{N}-X_{s}^{N}\right|<e$ and zero otherwise. The correlation integral, $C_{N}(e)$, can be interpreted as an estimate of the probability that $X_{t}^{N}$ and $X_{s}^{N}$ are within a distance $e$. Given this interpretation, we can see that under the independence hypothesis

$$
C_{N}(e) \longrightarrow C_{1}(e)^{N}, \text { as } T \longrightarrow \infty
$$

holds. That is, $P\left(\left|X_{t+i}-X_{s+i}\right|<e\right),(i=0,1, \ldots, N-1)$ is, due to independence, equal to $\prod_{i=1}^{N-1} P\left(\left|X_{t+i}-X_{s+i}\right|<e\right)$, which is estimated by $C_{1}(e)^{N}$ as the variables are identically distributed (Brock,et al., 1991 and Chappell, et al., 1996). Thus, the BDS statistic reduces to

$$
W_{N}(e)=\left[\sqrt{T}\left(C_{N}(e)-C_{1}(e)^{N}\right] / \hat{\sigma}_{N}(e),\right.
$$

where $\hat{\sigma}_{N}(e)$ is an estimate of the standard devision under the null hypothesis. The distribution of $W_{N}(e)$ converges to a standard normal with expectation zero and a variance unity, as $T$ approaches infinity. Thus, one can now calculate the statistic that has a standard normal asymptotic distribution under the independence hypothesis. If the absolute values of the test statistic are large, the null hypothesis of IID (randomness) is to be rejected. The critical values reported by Brock, et al.(1991) for significance levels of 0.05 and 0.01 are 2.22 and 3.40 respectively. 
Table 1. The Results of the BDS Test

\begin{tabular}{|rr|r|}
\hline$e$ & $N$ & \multicolumn{1}{|c|}{$W$} \\
\hline 0.1 & 2 & 22.375 \\
& 3 & 28.354 \\
& 4 & 48.363 \\
& 5 & 63.306 \\
& 6 & 85.462 \\
& 7 & 114.240 \\
& 8 & 146.171 \\
& 9 & 201.328 \\
& 10 & 275.680 \\
& 20 & 0.519 \\
\hline 0.5 & 2 & 10.070 \\
& 3 & 9.628 \\
& 4 & 9.538 \\
& 5 & 9.334 \\
& 6 & 9.030 \\
& 7 & 8.906 \\
& 8 & 8.774 \\
& 9 & 8.325 \\
& 10 & 8.051 \\
& 20 & 5.340 \\
\hline 0.9 & 2 & 26.710 \\
& 3 & 33.548 \\
& 4 & 44.931 \\
& 5 & 49.905 \\
6 & 54.972 \\
& 7 & 60.556 \\
& 8 & 65.792 \\
9 & 70.334 \\
& 10 & 76.659 \\
20 & 114.840 \\
\hline
\end{tabular}

\section{Obtaining the Experimental Signal}

The data were collected from the circuit shown in Figure 1. This circuit has only one nonlinear element the silicon diffused rectifier diode. We can model the diode as a nonlinear capacitor in parallel with a nonlinear resistor as discussed by Matsumoto (1987). The behavior of the circuit has been thoroughly studied in several papers during the last two decades (see Linsay, 1981 and Matsumoto, et al., 1984). Therefore, we will not go into details here. It has been rigorously proven that if the 
circuit parameters and the external drive (input voltage) are chosen appropriately, the system admits nonlinear oscillations.

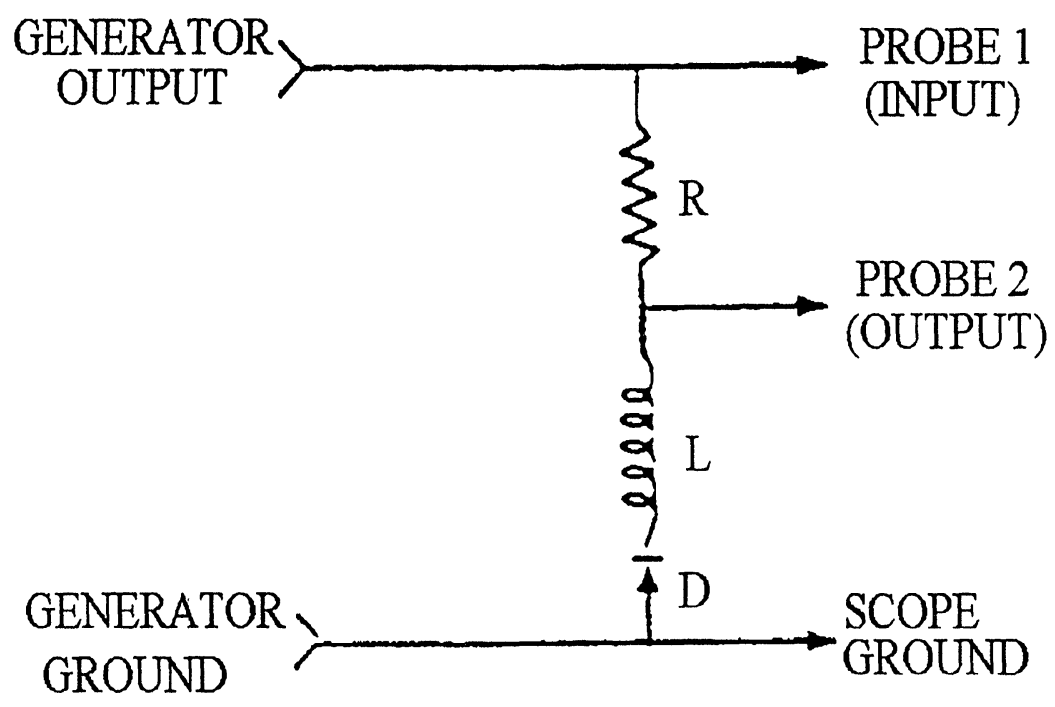

Figure 1. The diagram of circuit: $R=220 \Omega, L=2 m H, D=1 N 4001$

In our experiment, we used a 1N4001 silicon diffused rectifier diode, but any diode with a large capacitance can be used (Smith, 1992). The driven sinusoidal voltage was obtained from a function generator; the experimental results were obtained from an oscilloscope whose probes were attached as in Figure 1.

The nature of the output signal depends on the value of the input voltage. We have observed period doubling for (input voltage) Vin $=160 \mathrm{mV}$ and for $\mathrm{f}=80$ $\mathrm{kHz}$. After a number of period doublings, a chaotic signal was observed for Vin $=$ $120 \mathrm{mV}$ and $\mathrm{f}=6 \mathrm{kHz}$ as in Figure 2.

\section{Results of the Chaotic Analysis}

Chaotic analysis has been realised in two forms; analysis of the raw data and analysis of the first differenced series which was obtained from the $\left(X_{t}-X_{t-1}\right)$. In this paper, we will discuss only the results that belong to the first differenced data series in detail. Because, autocorrelation can affect some tests of chaos, so that we must remove it from data. This is typically done by taking the first difference of the data (Hsieh, 1991). The first differenced of the output data are plot in Figure 3. 


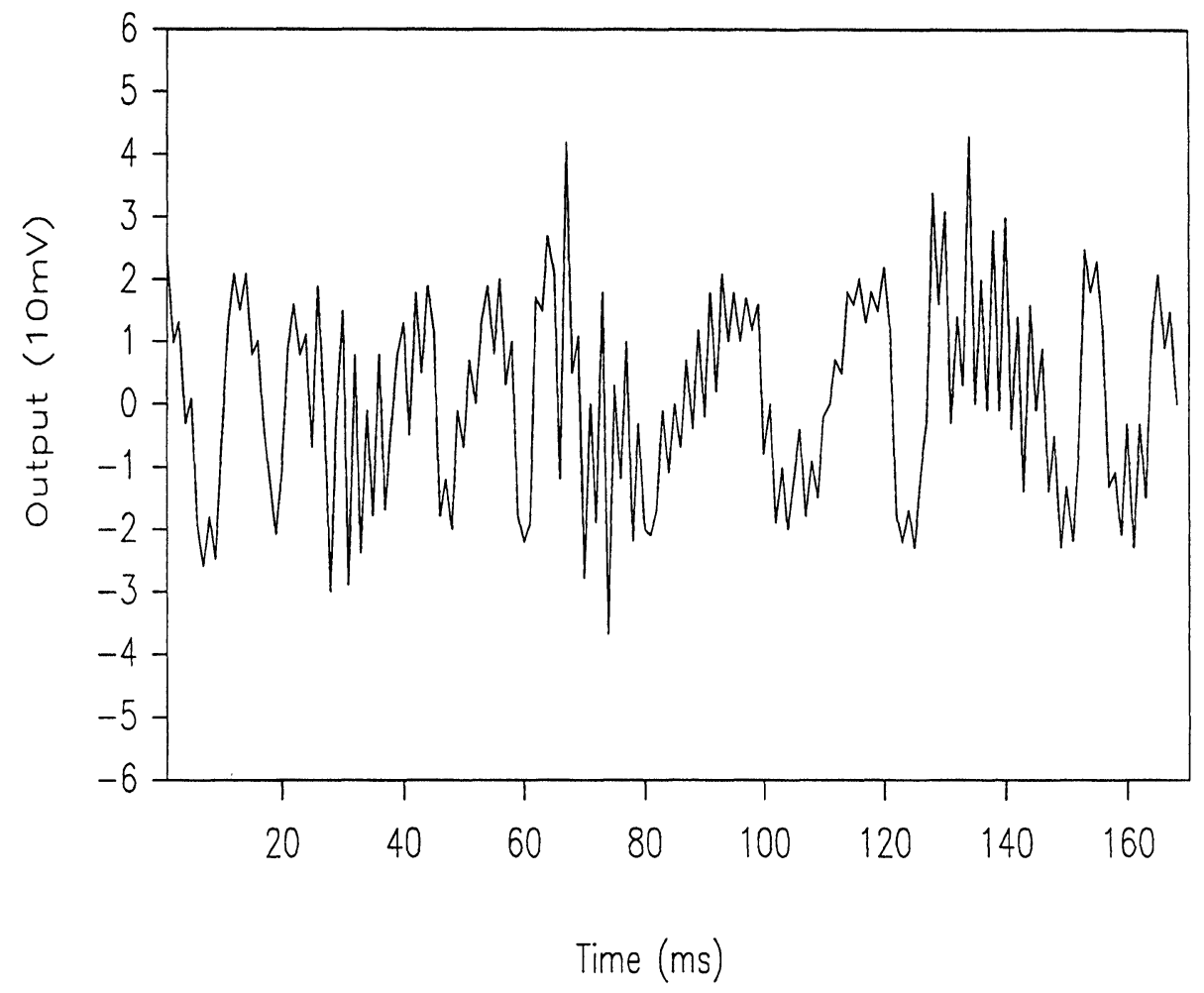

Figure 2. The output signal taken at $\mathrm{Vin}=120 \mathrm{mV}$ and $\mathrm{f}=6 \mathrm{kHz}$

Table 1 gives the the results of the BDS test applied to the first differenced data. The embedding dimension, $N$ is as varied from 2 to 10 and $e$ was chosen to be 0.1 , 0.5 and 0.9 for each series. Clearly, the BDS statistics lies within the positive tail of the standard normal distribution for these data. Hence we have rejected the null hypothesis that the data are IID. However, for a very high embedding dimension like 20 the null hypothesis can not be rejected. This result also supports that the data are chaotic.

\section{Conclusion}

We have investigated chaos using the BDS test statistic on an electrical signal taken from the RL-diode series connection. In an earlier study changing the embedding dimension $N$, from 2 to 10 and choosing $e$ to be $0.1,0.5$ or 0.9 indicated that the data manifested chaotic behavior for $e$ being 0.1 and 0.9 but not for $e$ being 0.5 . Results of this early analysis are not given in this paper, however, they can be obtained from the authors. 


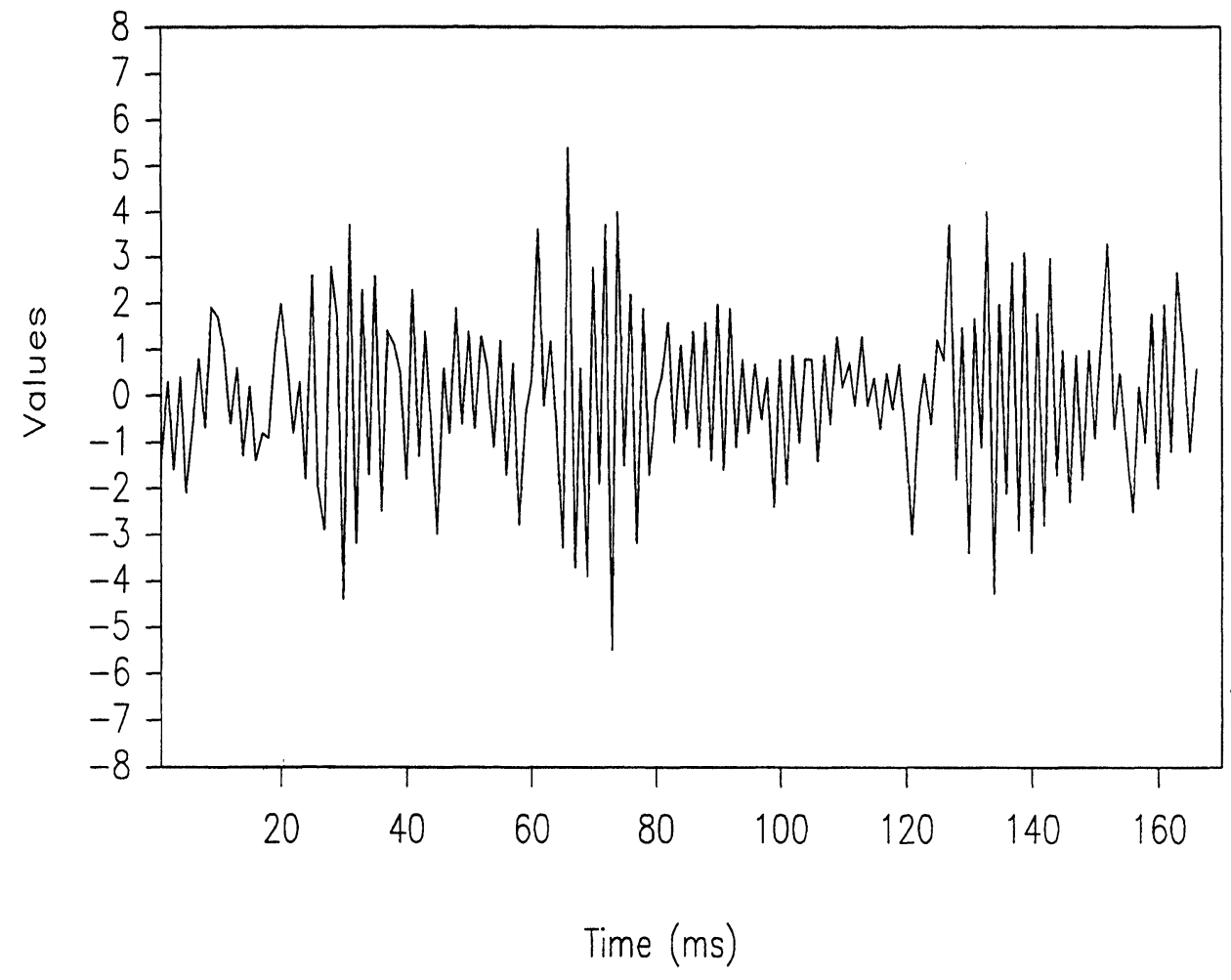

Figure 3. The plot of the first differenced series.

According to the results for the first differenced data, chaotic structure has been found for each $e$ values. These results indicate that chaotic structure in electrical circuits can be investigated by using BDS test.

\section{References}

1. W.A. Brock.; W.D. Dechert and J.A. Scheinkman. A test for independence based on the correlation dimension, Working Paper, University of Wisconsin, 1987.

2. W.A. Brock ; D.A. Hsieh and B. LeBaron. Non-linear Dynamics, Chaos, and Instability. Cambridge, Massachusetts: The MIT Press, USA, 1991.

3. D. Chappell ; J. Padmore and C. Ellis. A note on the distribution of BDS statistics for a real exchange rate series. Oxford Bulletin of Economics and Statistics, 58, 3, 561- 566, 1996.

4. J. Gleick. Chaos: Making a New Science. Viking Penguin, New York, 1987.

5. P. Grassberger and I. Procaccia. Measuring the strangeness of strange attractors. Physica, 9D, 189-208, 1983.

6. M.J. Hasler. Electrical circuits with chaotic behavior. Proceedings of the IEEE, 75, 1009$1021,1987$.

7. D.A. Hsieh. Testing for nonlinear dependence in foreign exchange rate, Journal of Business, 62, 3, 339-369, 1989. 
8. D.A. Hsieh. Chaos and nonlinear dynamics: an application to financial markets, Journal of Finance, 46, 5, 1839-1877, 1991.

9. J.P Keener and J.J. Tyson. Spiral waves in the Belousov-Zhabotinskii reaction. Physica D, 21, 307-324, 1986.

10. P.A. Linsay. Period doubling and chaotic behavior in a driven anharmonic oscillator. Phys. Rev. Lett., 47, 1349-1352, 1981.

11. T. Matsumoto. Chaos in electronic circuits. Proceeding of the IEEE, 75, 1033-157, 1987.

12. T. Matsumoto ; L.O. Chua and S. Tanaka. Simplest chaotic nonautonomous circuit. Phys. Rev., A30, 1155-1158, 1984.

13. T.C. Newell ; V. Kovanis ; A. Gavrielides and P. Bennett. Observation of the concurrent creation and annihilation of periodic orbits in a nonlinear RLC circuit. Phys. Rev., E54, 3581-3590, 1996.

14. J.A. Scheinkman and B. LeBaron. Nonlinear dynamics and stock returns, Journal of Business, 62, 3, 311-337, 1989.

15. D. Smith. How to generate chaos at home. Scientific American, January, 121-123, 1992. 


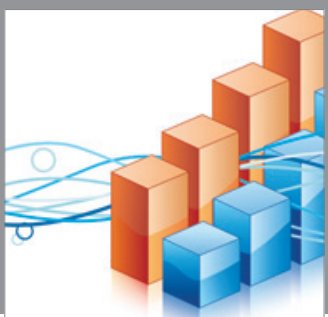

Advances in

Operations Research

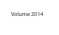

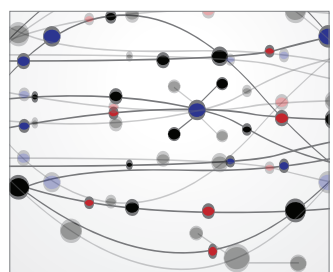

\section{The Scientific} World Journal
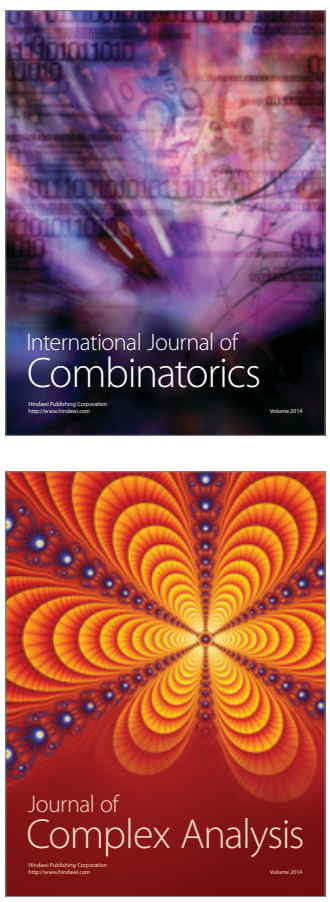

International Journal of

Mathematics and

Mathematical

Sciences
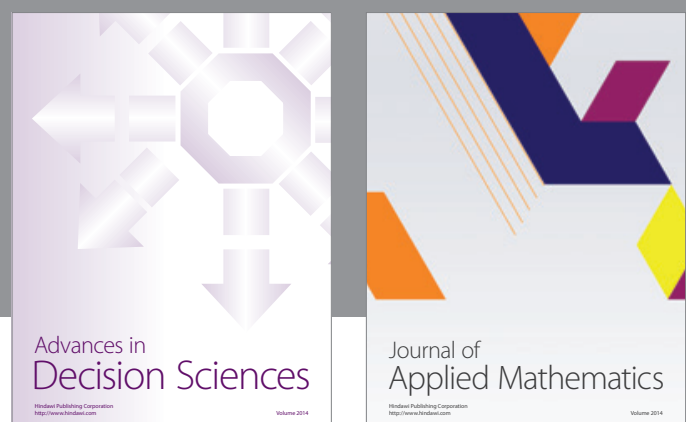

Journal of

Applied Mathematics
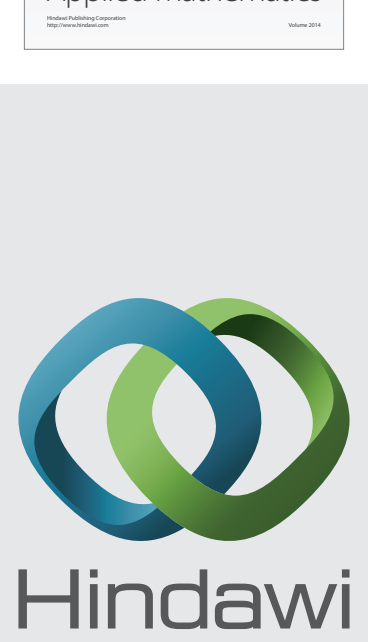

Submit your manuscripts at http://www.hindawi.com
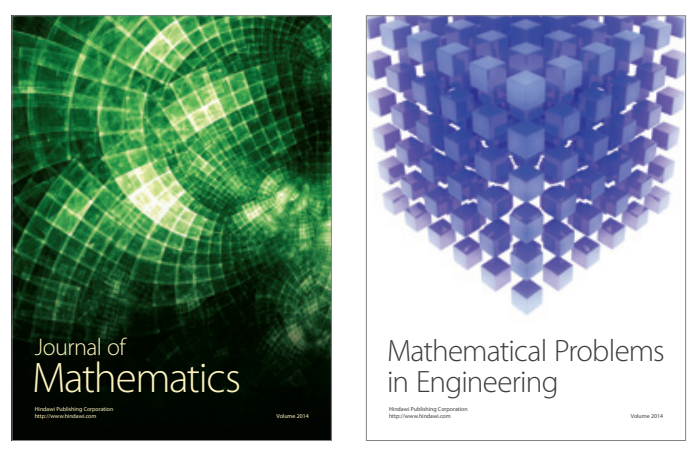

Mathematical Problems in Engineering
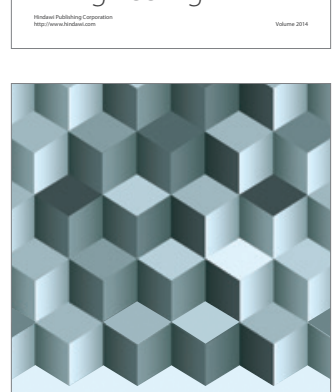

Journal of

Function Spaces
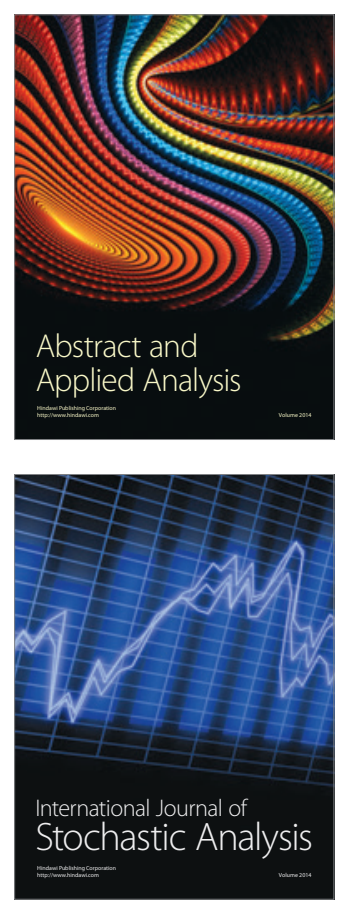

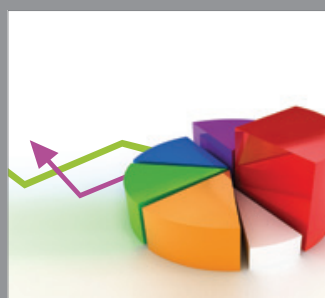

ournal of

Probability and Statistics

Promensencen
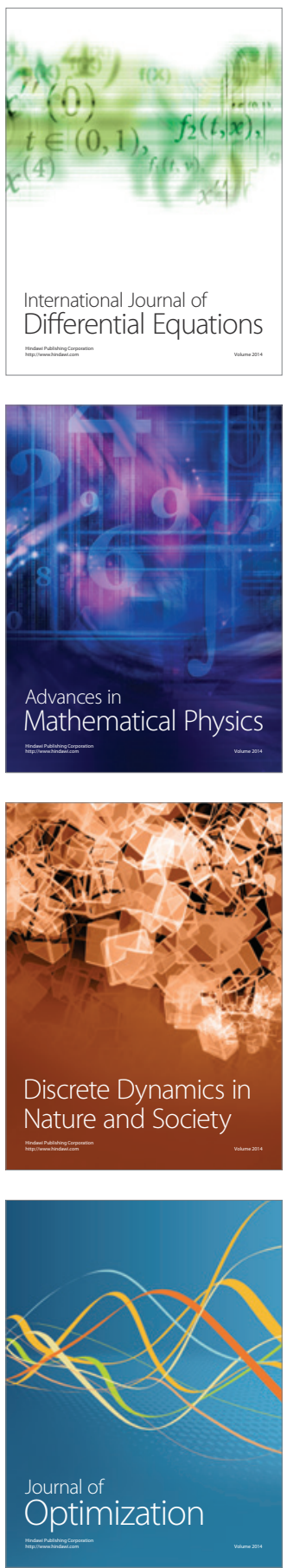